\title{
Mass gatherings and public health: preparing for World Youth Day 2008
}

\section{Jan Fizzell ${ }^{\mathrm{A}, \mathrm{B}}$, Sophie E. TynerC \\ and Jeremy M. McAnulty ${ }^{\mathrm{D}}$}

${ }^{\mathrm{A}} N S W$ Public Health Officer Training Program,

NSW Department of Health

${ }^{\mathrm{B} B i o p r e p a r e d n e s s ~ U n i t, ~ N S W ~ D e p a r t m e n t ~ o f ~ H e a l t h ~}$

${ }^{\mathrm{C}}$ Counter-Disaster Unit, Ambulance Service of NSW

${ }^{\mathrm{D} C o m m u n i c a b l e ~ D i s e a s e s}$ Branch, NSW Department of Health

This Bug Breakfast seminar was held prior to the World Youth Day activities in Sydney, New South Wales (NSW) in July 2008.

\section{Public health challenges and mass gatherings}

Public health preparedness for mass gatherings is essential for the delivery of a safe and healthy event. The World Health Organization recently defined mass gatherings as 'events attended by a sufficient number of people to strain the planning and response resources of a community, state or nation'. ${ }^{1}$

Public health challenges at mass gatherings include:

- the potential for communicable disease outbreaks

- mitigation of the risk of crowd crush

- the consideration of the likelihood of a terrorist event

- the likelihood of temperature-related illnesses (hypothermia and/or hyperthermia)

- sanitation

- events where active participation is encouraged (e.g. 'fun runs')

- access to safe food and water

- the possibility of crowd violence, noise issues, and the likely use and/or misuse of drugs and alcohol.

These challenges are compounded when events are of long duration, where little data relating to past experience with the event exists, and when protocols for managing the type of mass gathering are not readily available.

Some of the mitigation strategies that can be adopted include:

- rigorous pre-event planning

- regulation

- using appropriate health education strategies

- encouraging appropriate engineering to reduce the health risks to participants
- encouraging harm minimisation strategies

- encouraging pre-event vaccination

- pre-event surveillance and intelligence-gathering to better assess the risks of a particular event.

\section{Planning for World Youth Day 2008}

World Youth Day 2008 (WYD08) is a large-scale international gathering of Catholic youth which is taking place in July 2008. During 'Days in the Dioceses' from 10 to 14 July, participants can visit and undertake a program of activities at Diocese in locations across Australia and New Zealand. Participants will converge in Sydney for a program of major events, which is being held from 15 to 20 July. In Sydney, a large number of participants will stay in shared accommodation on the floors of school halls or commercial facilities.

Extensive public health planning has been undertaken for WYD08. This includes the formation of the WYD08 Public Health Working Party in September 2006, which has served as a conduit for early and regular engagement with key stakeholders including event organisers, laboratory services, local government and fellow government agencies. A public health project officer has been coordinating activities since November 2007, in order to assist all stakeholders in delivering a consistent response to WYD08.

There are significant communicable disease risks with an event of this type, especially considering the prolonged group contact and limited sanitation facilities available at temporary accommodation venues (such as school halls). To minimise the risks to participants, several strategies are being undertaken including encouraging pre-event vaccination and the provision of pre-event information encouraging hand washing and respiratory etiquette amongst pilgrims. Intra-event vaccination clinics may also be deployed (e.g. for Hepatitis A or varicella). A food preparation and inspection program has been developed by the NSW Food Authority, in consultation with local government. Advice has been provided to event organisers regarding the need to provide adequate hand washing facilities and isolation areas for sick participants.

The existing communicable disease surveillance program will be enhanced. Calls to a health advice line for participant accommodation team leaders and presentations to special event onsite medical units will be monitored for 
conditions of public health interest. The Australian Government Department of Health and Ageing will assist by monitoring for international events that may have an impact on WYD08. Cooperation has been sought from other jurisdictions regarding pre-event health surveillance. The NSW Department of Health and all area health service public health units will remain on high alert and ready to respond to outbreaks of disease during WYD08, using agreed protocols.

Mass gatherings present both challenges and opportunities. Many of the strategies developed for managing
WYD08 will be applicable in other public health emergencies, including mass evacuations and large-scale communicable disease outbreaks.

\section{Reference}

1. World Health Organization. Communicable disease alert and response for mass gatherings: key considerations, June 2008. Available from: http://www.who.int/csr/Mass_gatherings2.pdf (Cited 30 June 2008.) 